\title{
Iatrogenic Esophageal Rupture during Intragastric Balloon Insertion: A Case Report and Review of Literature
}

\author{
Mohamed Alassal*, Mohamed Elgazzar, Mohamed Saffan, Ibrahim Kasb, Ahmed Sobhy, Moataz Rizk, Bassim Mofreh, \\ Ashraf Elnahhas, Mahmoud Elshafea, Yousry Elsaed, Mohamed K Abdelshafi and Yousry Shaheen
}

Benha University Hospital, Faculty of Medicine, Cardiothoracic Surgery Department, Egypt

Received: 23 September, 2017; Accepted: 20 October, 2017; Published: 25 October, 2017

*Corresponding author: Mohamed Abdelwahab Alassal, Assistant professor and consultant of cardiothoracic surgery, Benha Faculty of medicine, Egypt, Email: dmohamedabdelwahab@gmail.com

\begin{abstract}
Iatrogenic esophageal traumas are the most common of esophageal perforation, accounting for up to $60 \%$ of cases from which endoscopic perforations are about $70 \%$. Despite modern surgical advanced techniques, perforation of the esophagus still represents a true surgical emergency and this condition is associated with a mortality of $20-30 \%$. Early and accurate diagnosis and treatment of esophageal injuries are critical. Indirect signs of esophageal injury can be seen on a posteroanterior and lateral plain chest X-ray. Such signs include pleural effusion, pneumomediastinum, subcutaneous emphysema, hydrothorax, pneumothorax and collapse of the lung. Computer Tomography (CT) of the chest and upper abdomen with oral contrast can also show a leak and confirm the chest X-ray findings. Three approaches are available for the treatment of esophageal perforation: conservative, endotherapy, and surgery. Surgery is mandatory in any part of the esophagus when the perforation is large or when patients do not improve with conservative or endoscopic treatment. We present our case who survived a massive thoracic esophageal tear during endoscopic intragstric balloon insertion. Emergency right thoracotomy was done; we found an inflated gastric balloon perforating the thoracic esophagus and protruding in the right thoracic cavity with a big longitudinal esophageal tear. The tear was primarily repaired with suture of perforation with reinforcement flaps. We conclude that; iatrogenic traumatic injuries of the esophagus is rare but could be life threatening and must be managed by multiple disciplinary teams. Urgent surgical esophageal repair is the treatment of choice in big thoracic esophageal tears. This is the 1st case documented with esophageal rupture due to intragastric balloon insertion.
\end{abstract}

\section{Introduction}

Iatrogenic causes are the most common of esophageal perforation, accounting for up to $60 \%$ of cases from which endoscopic perforations are about 70\% [1]. Other instrumentation modalities commonly include sclerotherapy, variceal ligation, pneumatic esophageal dilation, bougienage, and laser treatment. Placement of endotracheal, nasogastric, and Blakemore tubes represent less common iatrogenic causes [2]. Intraoperative esophageal perforation is a recognized complication of surgery, especially cardiothoracic or fundoplication, accounting for around $2 \%$ of all perforations. Other causes of traumatic esophageal injuries include blunt trauma like traffic accidents, penetrating traumas like stab wound and gunshot, foreign body swallowing like bone fish [3].
Spontaneous Esophageal perforations accounts about 15\% of all esophageal perforation and was first described in 1724 by Hermann Boerhaave. After autopsy he described the clinicpathologic correlation between esophageal perforation and a fatal outcome, one that remained inevitable for over 200 years [4].

Esophageal injury may be also classified as extraluminal or intraluminal. The extraluminal category includes processes originating outside the esophagus, including penetrating blunt trauma and surgery involving adjacent organs. The intraluminal classification acounts processes within the esophagus itself, including instrumentation, barotrauma, and foreign body ingestion [5].

Despite modern surgical advanced techniques, perforation of the esophagus still represents a true surgical emergency and this condition is associated with a mortality of $20-30 \%$, this is due to the anatomical location of the oesophagus which predisposes to mediastinitis which carries a high mortality $[6,7]$. Several factors, including the difficulty of accessing the esophagus, the lack of a strong serosal layer, the unusual blood supply of the organ and the proximity of vital structures, all contribute to this condition's high morbidity and mortality [8].

The gastric balloon procedure (endoscopic intra-gastric balloon) leaves an inflated silicon balloon in the stomach for 6 months, making less room for food and subsequently significant weight loss. Have health improvement for diabetes, joint/bone disease, and heart-related issues. These approved devices are placed via the esophagus using endoscopy. This can be done in an outpatient setting under sedation. Once in place the balloon is filled with saline and remains as a free floating object in the stomach cavity, too big to pass through the pylorus (Figure 1a, b). In addition to saline, the balloon that is made from silicone may contain some radio-opaque material as a radiographic marker and a dye such as methylene blue to alert the patient when the balloon would leak. It should not be applied to patients with certain intestinal problems such as inflammatory bowel disease or delayed gastric emptying, who are pregnant, or who are taking daily aspirin medication $[15,16]$.

We present here our case who survived a severe iatrogenic thoracic esophageal tear during intragstric balloon insertion. 


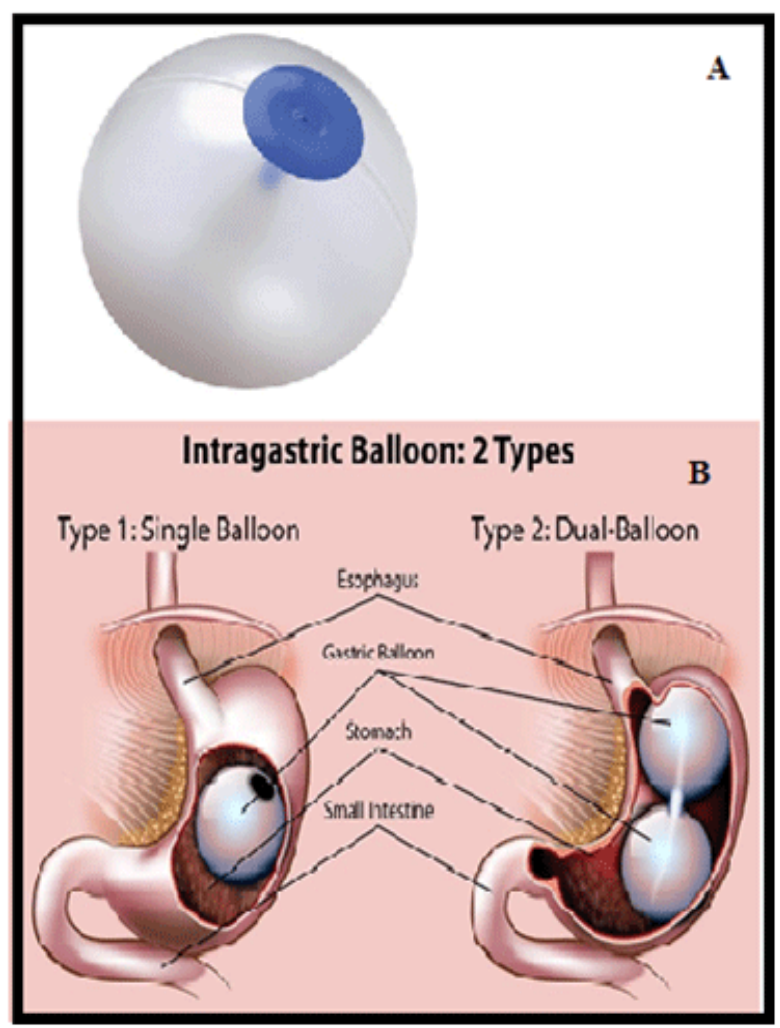

Figure 1 A,B: Types of intra-gastric balloon (Single and dual balloon)

\section{Case description}

We present our experience in a patient admitted to our cardiothoracic surgery department as a case of iatrogenic esophageal rupture. She is a 45 years old lady, well-known to have DM II, HTN, hyperlipidemia, morbid obesity, her BMI is $42 \mathrm{~kg} \backslash \mathrm{m} 2$; presented with severe chest pain and respiratory distress after a bariatric surgeon did for her an endoscopic single chamber Heliosphere BAG intragastric (Tourmed company) balloon insertion under general anesthesia in bariatric surgery department.

Immediate CXR and CT thorax done and revealed right pneumothorax and surgical emphysema in right chest wall with the radio-opaque big balloon in the right pleural cavity (Figure $2 \mathrm{a}, \mathrm{b})$. After consensus between the radiologist, gastroentrology surgeon, thoracic surgeon and bariatric surgeon, the decision was that; it is most likely an iatrogenic thoracic esophageal massive rupture and urgent thoracotomy for exploration and possible repair must be done.

After admission to ICU; resuscitation of the patient was done and right intercostals tube was inserted, then the patient was taken to our cardiothoracic surgery operating room and right thoracotomy was done then we found an inflated gastric balloon in the right thoracic cavity with a big longitudinal esophageal tear (Figure $3 \mathrm{a}, \mathrm{b}, \mathrm{c}$ ). The tear was Primary repaired with suturing of perforation with reinforcement flaps and the patient transferred to the ICU in a stable condition and with 2 right intercostals tubes (apical and basal tubes) and NG tube. She remained nearly NPO
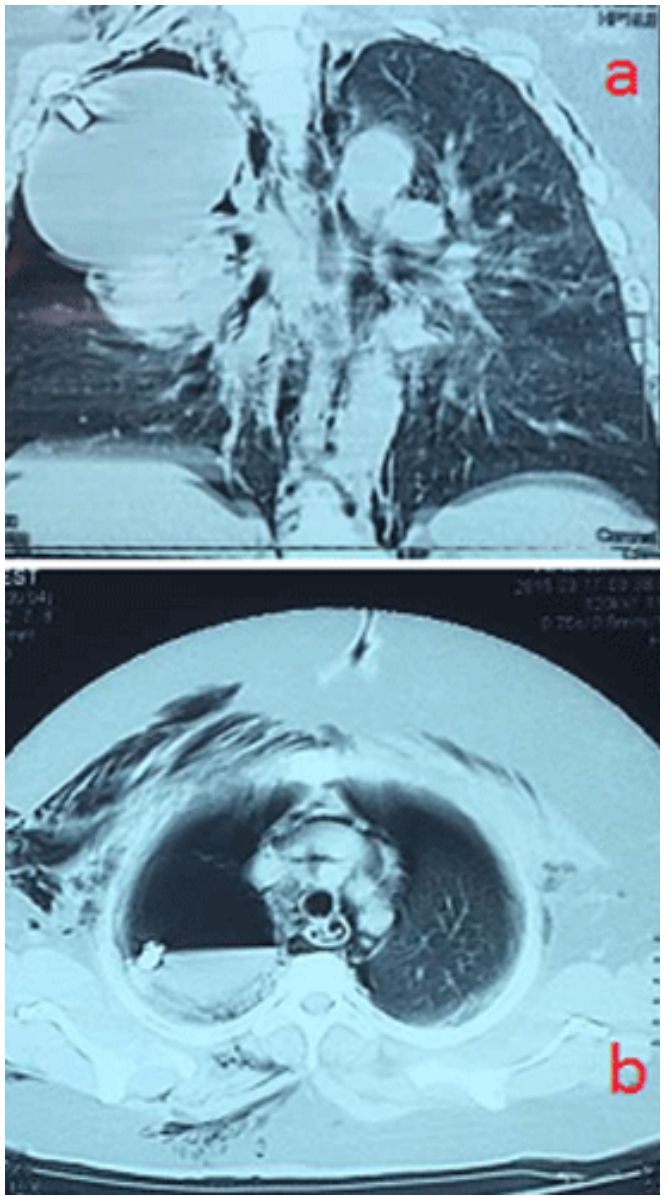

Figure 2 a,b: CT chest showing the inflated gastric balloon in the right pleural cavity with pneumothorax and surgical emphysema
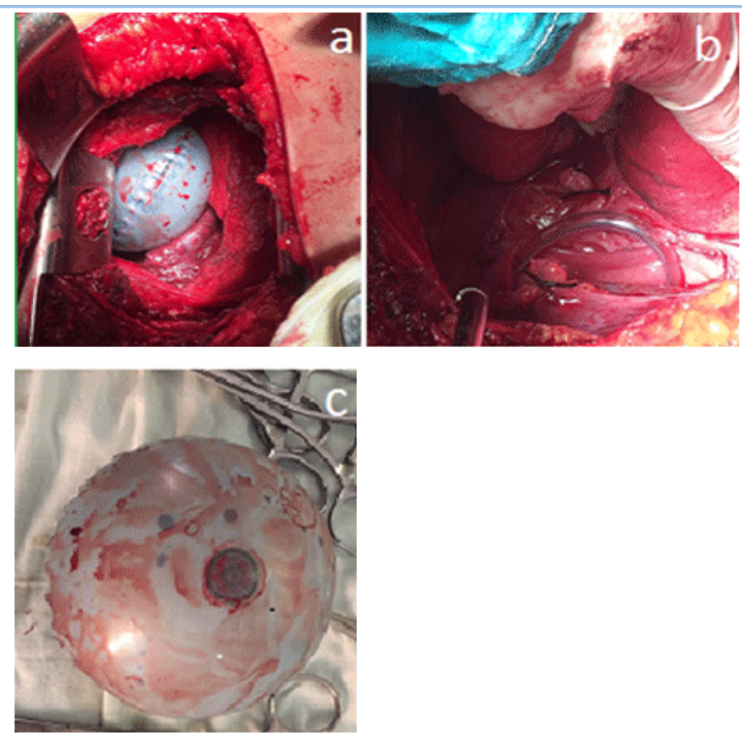

Figure 3: a) The gastric balloon in the chest cavity after thoracotomy b) Esophageal big longitudinal tear with NGT protruding out from the tear

c) The balloon after removal from the chest cavity 
for 10 days with TPN then gradually oral feeding was started and she tolerated the oral feeding well and discharged home 3 weeks after surgery in a good condition.

\section{Discussion}

Early and accurate diagnosis and treatment of esophageal injuries are critical, because clinical presentations are often vague and nonspecific and delayed diagnostic work-up has a negative effect on appropriate treatment and on the outcome [9] Diagnosis of an esophageal perforation relies on radiographic evidence. Specifically, indirect signs of esophageal injury can be seen on a postero-anterior and lateral plain chest X-ray. Such signs include pleural effusion, pneumomediastinum, subcutaneous emphysema, hydrothorax, pneumothorax and collapse of the lung [10]. However, a chest X-ray with water-soluble contrast medium (if the patient can swallow) reveals a contrast leak in most cases of esophageal perforation. Computer Tomography (CT) of the chest and upper abdomen with oral contrast can also show an esophageal leak. In addition, collection of air or fluid in the mediastinum, pleural effusions, pneumocardium and pneumoperitoneum are important diagnostic findings in these patients. The site of perforation and the degree of containment may be easier to judge by CT than by plain chest X-ray $[9,10]$ In a patient with high suspicion of perforation with negative radiography, or when swallowing a contrast agent is impossible for technical reasons, flexible endoscopy should be considered. This widely available tool allows direct visualization of the entire esophagus and stomach and may also provide additional information about the acute onset of symptoms in patients without previous instrumentation used in the esophagus [11].

In the mid-20th century, successful primary repair of esophageal perforation was first reported by Barrett in 1946 and later by Olsen and Claggett in 1947, and esophagectomy for esophageal perforation was described by Satinski and Kron in 1952 , all of which led the way to the modern treatment of these injuries $[7,12]$.

Three approaches are available for the treatment of esophageal perforation: conservative, endotherapy, and surgery. The location whatever cervical, thoracic, or abdominal portions of the esophagus and size of the perforation influence treatment choice. Cervical perforations are usually small and can be treated conservatively as the perforation or leak is also contained within the triangle of Killian in the neck. Most cervical perforations have a good outcome with conservative treatment with intravenous antibiotics and nil by mouth. Treatment of thoracic perforations depends very much on the size of the perforation. Small perforations due to sclerotherapy injection, for example, can be treated conservatively. Endotherapy can help avoid surgery in other cases: small tears from endoscopic insertion can be clipped and esophageal fistulae can be injected with fibrin glue. Larger perforations can be treated with stent placement if the dehiscence of the lumen circumference does not exceed 70\% [11, 12]. Perforation of the intra-abdominal portion of the esophagus often results in a rapid development of peritonitis and sepsis and surgery is usually recommended. Surgery is mandatory in any part of the esophagus when the perforation is large or when patients do not improve with conservative or endoscopic treatment. In very ill patients, esophageal exclusion surgery can be carried out until the patient's general condition stabilizes. In cases of a diseased esophagus such as corrosive injury related perforations or cancer of the esophagus, esophageal replacement surgery should be contemplated with total esophagectomy and gastric pull-up surgery or creation of a neoesophagus with colonic interposition $[12,13]$.

The gastric balloon or intragastric balloon is an inflatable medical device that is temporarily placed into the stomach to reduce weight. The history of intragastric balloons (IGBs) began in 1985 with the Garren-Edwards Bubble. It was approved by the U.S. Food and Drug Administration (FDA) for temporary use as a weight loss device, but its manufacture was discontinued in 1988, and approval was withdrawn in 1992 because of significant complications and limited weight loss. A number of IGBs have appeared since that time, mostly originating in Europe or South America, but none has acquired FDA approval until recently; the ReShape Duo Integrated Dual Balloon System (ReShape Medical Inc., San Clemente, California) received FDA approval in August $2015[14,15]$.

We presented our case who survived a severe iatrogenic thoracic esophageal tear during intragstric balloon insertion and we think that the rapid accurate diagnosis and urgent surgical management was very crucial in this case.

\section{Conclusion}

We conclude that; iatrogenic traumatic injuries of the esophagus are rare, but life-threatening and should be suspected in any case with vague symptoms post endoscopy. Despite esophageal perforations is rare, clinicians in multiple disciplines, including general surgery, emergency medicine, thoracic surgery, trauma surgery, otolaryngology, and spine surgery must be aware and oriented regarding their diagnosis and management. Urgent primary surgical esophageal repair is the treatment of choice in large thoracic esophageal tears. We think that our case is unique because up to our knowledge this is the 1st documented case with thoracic esophageal rupture due to intragastric balloon insertion and inflation. In our patient, the diagnosis of the esophageal perforation was made early and a multidisciplinary team chose urgent surgical repair as the treatment of choice given that the patient was not septic and had no contraindications to the surgery. This was instituted without complications, and achieving good results.

\section{References}

1. Ugo A Ezenkwele, Steven C Dronen and Corey M Long. Esophageal Rupture and Tears in Emergency Medicine Treatment \& Management. Drugs \& Diseases; Emergency Medicine. 2016.

2. Peter Waweru, David Mwaniki. Conservative Management of an Iatrogenic Esophageal Tear in Kenya. Case Rep Surg. 2015. doi: $10.1155 / 2015 / 102540$

3. Hasan S, Jilaihawi AN, Prakash D. Conservative management of iatrogenic oesophageal perforations - a viable option. Eur J Cardiothorac Surg. 2005;28(1):7-10.

4. Curci JJ, Horman MJ. Boerhaave's syndrome: The importance of early diagnosis and treatment. Ann Surg. 1976;183(4):401-418. 
5. Kavic SM, Basson MD. Complications of endoscopy. Am J Surg. 2001;181(4):319-332.

6. Ronald V Romero, Khean-LeeGoh. Esophageal perforation: Continuing challenge to treatment. Gastrointestinal Intervention. 2013;2(1)1-6. doi.org/10.1016/j.gii.2013.02.002

7. Cooke DT, Lau CL. Primary repair of esophageal perforation Operative Techniques in Thoracic and Cardiovascular Surgery. 2008; 13(2):126-137.

8. Rachna Madan, Ryan James Bair, Jeffrey Forris Beecham Chick Complex Iatrogenic Esophageal Injuries: An Imaging Spectrum. American Journal of Roentgenology. 2015;204(2).

9. Soreide JA, Viste A. Esophageal perforation: diagnostic work-up and clinical decision-making in the first 24 hours. Scand J Trauma Resusc Emerg Med. 2011;19:66. doi: 10.1186/1757-7241-19-66

10. Shaker H, Elsayed H, Whittle I, Hussein S, Shackcloth M. The influence of the 'golden 24-h rule' on the prognosis of oesophageal perforation in the modern era. Eur J Cardiothorac Surg. 2010;38(2):216-222. doi: 10.1016/j.ejcts.2010.01.030
11. John J Gleysteen. A history of intragastric balloons. Surgery for Obesity and Related Diseases. 2016;12(2):430-435. doi. org/10.1016/j.soard.2015.10.074

12. Helton WB, Unnikrishnan R, Gal T. Cervical esophageal perforation and cricopharyngeal dysfunction. Ear Nose Throat J. 2011;90(3):E8E10.

13. Vogel SB, Rout WR, Martin TD, Abbitt PL. Esophageal perforation in adults: aggressive, conservative treatment lowers morbidity and mortality. Ann Surg. 2005;241(6):1016-1021.

14. Freeman RK, Herrera A, Ascioti AJ, Dake M, Mahidhara RS. A propensity-matched comparison of cost and outcomes after esophageal stent placement or primary surgical repair for iatrogenic esophageal perforation. J Thorac Cardiovasc Surg. 2015;149(6):1550-1555. doi: 10.1016/j.jtcvs.2015.01.066

15. VafaShayani. Gastric Balloon - 14 ways it will affect you. Bariatric Surgery Source. 2017

16. Buzga M, Evzen M, Pavel K, Tomas K, Vladislava Z, Pavel Z, et al. Effects of the intragastric balloon MedSil on weight loss, fat tissue, lipid metabolism, and hormones involved in energy balance. Obes Surg. 2014;24(6):909-915. doi: 10.1007/s11695-014-1191-4 